\title{
SIMULATION OF CRACK PROPAGATION/DEFLECTION IN CERAMIC MATRIX CONTINUOUS FIBER REINFORCED COMPOSITES WITH WEAK INTERPHASE VIA THE EXTENDED FINITE ELEMENT METHOD
}

\author{
M. Braginsky ${ }^{a, b}$ and C. P. Przybyla ${ }^{a^{*}}$ \\ ${ }^{a}$ Materials and Manufacturing Directorate, Air Force Research Laboratory, Wright-Patterson AFB, Ohio, \\ USA \\ ${ }^{b}$ University of Dayton Research Institute, Dayton, Ohio, USA \\ *Corresponding Author: craig.przybyla@us.af.mil
}

Keywords: Crack Deflection, Ceramic Matrix Composites, XFEM, Fracture.

\begin{abstract}
Toughness in continuous ceramic fiber reinforced ceramic matrix composites (CMCs) with dense matrices depends on the properties of the fiber coating or interphase. Multiple criteria have been proposed to describe the mechanism of crack propagation/deflection at the filament scale in brittle matrix continuous fiber reinforced composites; however, most of these criteria fail to account for the presence of an interphase of finite thickness and/or employ unrealistic boundary conditions. Recent simulations employing the extended finite element method (XFEM) have shown that variations in interphase thickness and strength relative to the fibers and/or matrix can have a significant influence on the crack propagation/deflection mechanism. It is shown that primary crack deflection most often occurs when conditions favor secondary cracking in the interphase in front of an approaching matrix crack. Although this mechanism is similar to that argued by Cook and Gordon (Cook J, Gordon JE, Proc. Roy. Soc. A 1964; 28; 508-520), the simulations here indicate that the conditions for secondary crack initiation and deflection of the primary crack can be much different than that which was originally presented in their analytical model. Variations in the properties of the interphase are simulated to produce large deviations in the local crack growth behavior as a matrix crack grows into interphase. Results are discussed relative to what has been observed experimentally.
\end{abstract}

\section{Introduction}

Toughness in continuous ceramic fiber reinforced ceramic matrix composites (CMCs) is enabled through one of two mechanisms. In CMCs with a porous matrix, cracks in the matrix lack a stress singularity, and propagate by the means of the sequential failure of the cell walls surrounding the pores when the load is of sufficient magnitude. In this manner, fibers are not subject to a stress singularity from an approaching matrix crack and resist failure due to their superior strength relative to the weaker matrix. The second mechanism is observed in the presence of a fairly dense 
matrix when there is a weak interface or interphase (i.e., fiber coating) separating the fibers from the matrix. As matrix cracks propagate through the dense matrix they are deflected around the fiber by the weak interface and/or interphase surrounding the fiber. It is this latter mechanism that will be the focus of this work.

Deflection of matrix cracks at the interphase depends on the relative strengths of the matrix, interphase and fiber and the bonding characteristics between the constituent layers. Changes in the interphase strength and the bonding characteristics between the interphase and the fiber were shown by Rebillat et al. [1] to have the potential to double the overall tensile strength of a $\mathrm{SiC} / \mathrm{SiC} \mathrm{CMC}$ with a BN interphase. Optimization of composite strength and toughness in CMCs requires a detailed understanding of the fracture process at the fiber-matrix interface. Several approaches have been proposed to estimate the conditions of crack propagation versus deflection at matrix, interphase or fiber boundary. A often cited approach proposed by He and Hutchinson [2] employs traditional fracture mechanics to define a criterion for crack deflection at the fibermatrix interface in an isotropic material according to

$\frac{G_{d}}{G_{p}}<\frac{\Gamma_{i}}{\Gamma_{f}}$

where $G_{d}$ and $G_{p}$ are the energy release rates for deflection and propagation and $\Gamma_{f}$ and $\Gamma_{i}$ are the critical energy release rates or surface energies for the fiber and for a deflecting crack at interface between two semi-infinite planes with plane strain traction free boundary conditions. Later by including the effect of anisotropy, Martinez and Gupta [3] demonstrated that the energy for a doubly deflected crack is higher than that for a singly deflected crack in certain cases. Subsequently, $\mathrm{He}$ at al. [4] corrected their result for the doubly deflected crack and also considered the influence of residual stress. Similar results were shown numerically by Tullock et al. [5]. Also, Ahn et al. [6] numerically modeled the influence of crack extensions according to the criteria defined in Equation (1) and predicted that crack deflection requires more energy for finite crack extension and finite fiber volume fraction than previously shown in the limit of zero fiber volume fraction or infinitesimal crack extension as calculated analytically by $\mathrm{He}$ et al. [4]. Martin et al. [7] employed a quasi-static approximation for a propagating crack towards the interface under constant loading to avoid assumptions regarding the extension ratio that was employed by $\mathrm{He}$ and Hutchinson [4]. This modified approach with plane strain boundary conditions predicts deflection to be less favorable than what was shown by the $\mathrm{He}$ and Hutchinson [4] criterion when the crack lies in the stiffer material. The same energy release rate approach was applied to a related problem of cracks kinking out of an interface starting in He et al [8]. In particular, Xie et al in [9] and [10] used Virtual Crack Closure technique to partition strain energy release rates for individual fracture modes to numerically study the kinking criteria comparing these results to experiments in [10] and to the work of He et al [8].

None of the previously discussed approaches for crack deflection consider an interphase of finite thickness. A few studies have demonstrated that a finite interphase can have a significant influence on the overall response. For example, Martin et al. [11] employed fracture mechanics implemented in a finite element model to consider deflection with and without a finite interphase. In the absence of an interphase, it was predicted that crack deflection is enhanced if the toughness of the matrix is less than that of the fiber. Previous studies neglected any toughness mismatch between the fiber and matrix. In the presence of an interphase, enhanced crack deflection is 
predicted when the toughness at the fiber-interphase interface is low. Simulations by Parthasarathy et al. [12] employed the FEM to explicitly model the matrix, interphase and fiber to determine the optimal thickness of the interphase for crack deflection. Employing plane strain boundary conditions, it was predicted that a thin interlayer with low modulus may significantly enhance crack deflection, which supports what was calculated previously. Liu and Yang [13] employed a virtual crack closure technique implemented via the FEM to account for the influence of thermomechanical coupling between the matrix, interphase and fiber. They predict enhanced crack deflection when the thermal mismatch is larger between the matrix and interphase.

In all cases, the previously cited approaches account for one single dominant crack intersecting the fiber-matrix or matrix-interphase interface. However, secondary debonding or cracking at the interphase or at interphase boundaries in many materials is observed at some distance in front of the primary crack; this secondary crack arrests or retards growth of the primary crack [14-17]. Cook and Gordon [18] postulated this type of crack deflection mechanism where interfacial debonding was predicted ahead of the crack tip as a result of the stress component parallel to the crack plane, $\sigma_{r r}$, if the interface is sufficiently weak. They predicted that, for an elliptical crack, $\sigma_{r r}$ is maximum at a distance ahead of the crack tip on the order of the crack tip radius. Later Pagano and Brown [15] simulated cracking with and without the secondary cracking or interfacial debonding mechanism and predicted much higher energy release rates in the case of debonding of the interface ahead of the crack tip. Leguillon et al. [19] employed a asymptotic analyses to account for the influence of debonding at the interface ahead of the primary matrix crack. They showed that crack deflection is more favorable in the presence of a secondary crack initiated ahead of the primary crack than in the case were no secondary initiation occurs. Later Lacroix et al. [20] extended the previous analysis to establish an energy based criterion based on secondary crack initiation. Martin and Leguillon [21] then employed a similar approach with an axisymmetric fiber/matrix model loaded in tension. For a propagating crack, decohesion is always predicted when the fiber is stiffer than the matrix; whereas, in the case of a stiffer matrix, decohesion is unlikely. In another approach, Pompidou and Lamon [22] employed the Cook and Gordon model [18] to estimate the propensity for the interface ahead of the crack tip to debond based on the elastic mismatch and relative strengths of the fiber and matrix. However, the Cook and Gordon model [18] assumes an elliptical crack with finite radius and experimentally matrix cracks have been observed to be quite sharp. Stresses at crack tips of sharp cracks are very different than those of elliptical cracks. Additionally, cracks perpendicular to the crack plane can initiate in the interphase further ahead of the crack tip than where $\sigma_{r r}$ is maximum depending on the strength of the interphase, bonding between the matrix and interphase or interphase and fiber.

The effort described here employs the extended finite element method XFEM [23-25] implemented in ABAQUS $^{\circledR}$ [26] to study fiber/matrix crack deflection in SiC/SiC CMC with a BN fiber interphase. In all the previous studies cited, the cracks and/or crack propagation were defined a priori. Employing the XFEM here allow the simulation of crack initiation and growth without predefinition. Some of the results here have been describe previously [27, 28]. Different mechanisms of crack progression/deflection can be observed depending on the relative strengths of the matrix, interphase, and fiber and applied boundary conditions. In this work both 2D plane strain and axisymmetric boundary conditions are employed. The 2D plane conditions are similar to many of modeling approaches cited previously including the original work of $\mathrm{He}$ and Hutchinson [2]. However, as it will be shown here via simulation the boundary conditions can significantly affect the operant mechanisms for crack deflection as well as the relative strength of 
the constituent properties. Material properties of a commercially produced $\mathrm{SiC} / \mathrm{SiC} \mathrm{CMC}$ were taken as the basis for the simulations, but different ranges of these properties were considered to understand the impact of relative strengths on the operant mechanisms. Reduced integration was not employed in any of the simulations. The fracture energy of the interface between the matrix/interphase and interphase/fiber was taken to be the same as the interphase (the weakest phase).

\section{Simulation methodology}

A representative $\mathrm{SiC} / \mathrm{SiC} \mathrm{CMC}$ based on the HiPerComp® material produced by GE Aviation ${ }^{\circledR}$ through the melt-infiltrated processed was explicitly modeled with the strength of the matrix being less than that of the fiber as shown experimentally. Basic properties of the fiber (SiC), matrix $(\mathrm{SiC})$ and fiber coating or interphase $(\mathrm{BN})$ were assumed based on experimental results [29]. The thickness of the interphase was assumed to be $1 \mu \mathrm{m}$. The material properties employed in the simulations are summarized in Table 1.

To evaluate the influence of damage initiation and propagation parameters on the behavior of the system, a range of strengths was considered for the matrix and the interphases. The range of properties for the 3D axisymmetric simulations was slightly more extensive then the conditions considered for the 2D plane strain boundary conditions because the types of mechanisms observed in axisymmetric simulations was more plentiful. The range of values considered for the simulations for both types of boundary conditions are given in Table 2.

An initial crack was incorporated into the model to study crack propagation characteristics as the cracks grow up to the fiber/matrix interphase. Loading in all the simulations was strain-controlled with uniform displacements applied at the boundaries parallel to the initial crack. The remaining boundary conditions were assumed to be traction free. Damage initiation is predicted based on a maximum principal stress initiation criterion, and values of this initiation stress (Tables 1 and 2) are referred to as the strength. Crack evolution is governed by cohesive laws where the fracture energy defines the rate at which cohesive stiffness is degraded once the initiation criterion is met.

The commercially available FEA software package ABAQUS [30] was employed for all of the simulations. Loading was quasi-static, and viscous regularization was employed to aid convergence of the solution at each integration time step. In ABAQUS Standard (i.e., with implicit integration), the viscous regularization is controlled by the parameter damage stabilization. The additional parameter tolerance controls incrementation of the step time. It is important to note that the implementation of XFEM in the version of ABAQUS employed here does not allow for crack coalescence. This is a major limitation when multiple cracks attempt to interact and coalesce. Instead of coalescence, the two cracks will continue to propagate unnaturally while avoiding each other and the resulting solution will be artificially stiff. The elements between the cracks that would like to coalesce remain unbroken and intact because crack coalescence is not numerically possible. In the simulations performed here where crack coalescence was judged to be necessary, results of the simulations were only considered up to the point where the cracks grew to within 2 elements of each other just prior to pending crack coalescence. 


\section{Results and Discussion}

\subsection{Simulations setup}

Figures 1 and 2 describe the simulated geometry and boundary conditions for the 2D plane strain and 3D axisymmetric simulations, respectively. The materials properties employed for both the 2D plane strain and 3D axisymmetric simulations are given in Table 2. There were pronounced differences in the results, with the 3D axisymmetric simulations exhibiting more variety in crack configurations as discussed below. However, generally the results of both types of simulations can be categorized into one of two cases, namely:

1. Initiation of single secondary crack in the interphase when the primary crack is far from the interphase/coating interface (i.e., on the order of several times the interphase thickness).

2. Multiple secondary crack initiation above and below the plane of the primary crack in the interphase when the primary crack has intersected the interphase/coating interface or is very close to the interphase (i.e., closer than the distance of $1 / 3$ the interphase thickness).

\subsection{Case 1: Secondary Crack Initiation in the Interface when Primary Crack is Far Away}

\subsubsection{D Plane Strain Simulations}

The crack patterns observed in 2D simulations are shown in Figure 3. Case 1 is hypothesized to be conducive to crack deflection because the initiation and growth of the secondary crack inhibits further growth of the primary crack upon coalescence of the primary crack with the secondary crack. In the simulations performed here, the operant mechanism of deflection in Case 1 is characterized by the initiation of a secondary crack near the interphase/matrix boundary when the primary crack's tip is at a finite distance (i.e., several widths of the interphase thickness) from the interphase with subsequent growth of this secondary crack into the matrix as shown in Figure 3(a). This predicted mechanism is not captured by the Hu-Hutchinson [2, 4] model with similar boundary conditions and is oversimplified by the model of Cook and Gordon [18] or Pompidou and Lamon [22]. Here it was predicted that a secondary crack can initiate in the interphase even when the approaching crack tip is located at a distance from the interphase/matrix boundary much greater than the radius of the crack tip. This contradicts Cook and Gordon's result that the influence of the crack in the matrix is limited to a process zone on the order of the crack tip radius [18].

The distinctive chevron cracks observed in Figure 3-(a) is predicted based on the maximum principle stress crack initiation criterion employed in these simulations. Similar en echelon cracking has been observed experimentally [31-34]. In these experimental observations, this cracking mechanism was observed to be localized within the interphase and did not propagate into the matrix. In contrast, for the simulations performed here, the secondary crack propagated into the matrix when the applied strain exceeded a certain magnitude. We note that the simulated volumes were quite constrained and that the applied boundary conditions of these 2D plain strain simulations are not representative of what actually occurs in 3D. It is also possible that the crack initiation and propagation criterion of max principle stress is not representative of the actual failure mode. Shear dominated failure modes could produce an alternate response. It is noted that 
the en echelon cracking observed experimentally was largely attributed to a Mode II type shear mechanism. Regardless, we consider the mechanisms observed here relative to the insight that can be gained from the relative properties for the applied boundary conditions and maximum principle stress failure criterion.

\subsubsection{D Axisymmetric Boundary Conditions}

When axisymmetric boundary conditions are imposed as depicted schematically in Figure 2, an increased variety of scenarios can be observed. The observed cracking patterns for the various simulated cases can be seen in Figures 4 and 5 for an interphase fracture energy of $5 \mathrm{~J} / \mathrm{m}^{2}$ and 15 $\mathrm{J} / \mathrm{m}^{2}$, respectively. Similar results were observed with the other simulated interphase fracture energies listed in Table 2. As seen in Figures 4 and 5 the cracking patterns are more varied - the secondary crack/cracks in the interphase can now appear not only at the coating/matrix interface, but also at the fiber/coating interface, for instance - but they still fall into the general categories (a) and (b) described above.

These competing modes are a result of the differences between the stress states in the interphase when the primary crack tip is removed some finite distance from the interphase-matrix boundary (as shown in Figure 6) on the order of several times the interphase thickness versus when the crack tip has intersected the interphase-matrix boundary (as shown in Figure 7). When the primary crack tip is at some distance from the interphase, the normal stress in the radial direction, $\sigma_{11}$ in Figure 6, dominates the stress field in the interphase. Secondary crack initiation occurs under these conditions when the coating failure criterion is met and results in the formation of a single secondary crack nearly parallel to the interphase/matrix interface. This secondary cracking appeared to favor conditions for crack deflection as will be discussed below.

\subsection{Case 2: Multiple Cracking in the Interphase upon Intersection of the Primary Crack with the Matrix-Interphase Boundary}

\subsubsection{D Plane Strain Simulations}

Crack penetration of the primary crack through the interphase/matrix interface and into the fiber is shown in Figure 3-(b). In this case, the primary crack propagates to the fiber boundary, pauses while the load continues to increase and then continues to propagate through the fiber. Secondary cracking or fragmentation (i.e., multiple secondary cracking) is observed in the interphase/matrix interface above and below the primary crack when the primary crack passes into the interphase. Secondary fragmentation similar to what has been simulated has not necessarily been observed experimentally; however, composites with these types of strong fiber coatings are not desirable. In fact, processing of CMCs with a strong interphase is avoided because they lack toughness. It is not clear if this simulated fragmentation is representative of reality or an artifact of the this particular implementation of the XFEM where multiple cracks in neighboring elements cannot be realistically captured due to the numerical limitations previously discussed. Despite these concerns, the simulations give insight into the anticipated behavior given the stiff constraints imposed by the 3D plain strain boundary conditions relative to the strength values for through fiber crack penetration based on the maximum principle stress initiation criterion that was employed here.

\subsubsection{D Axisymmetric Boundary Conditions}


Similar results are observed in simulations with the 3D axisymmetric boundary conditions as were observed in the simulations with applied 2D plane strain boundary conditions. When the crack tip intersects the interphase-matrix boundary as shown in Figure 7 the stress localizes at the primary crack tip at the interface boundary. In this case, the maximum principal stress drives multiple secondary crack initiation above and below the plane of the primary crack. In most cases when the primary crack intersects the interphase-matrix boundary before secondary crack initiation occurs, it is observed that the primary crack continues to grow and penetrate into the fiber resulting in complete failure of the composite. Multiple secondary crack initiation above and below the plane of the primary crack does not promote deflection of the primary crack.

\subsection{Discussion}

Various parameters were considered here to quantify the propensity of primary crack deflection versus crack penetration into the fiber for these simulations with $3 \mathrm{D}$ axisymmetric boundary conditions. Two parameters that had significant correlation to crack deflection versus penetration include both the distance of the primary crack tip to the interphase/matrix boundary at the point of secondary crack initiation and the angle of the maximum principal stress relative to the interphase/matrix interface along the plane of the primary crack at the interphase/matrix interface. These two parameters are hypothesized to be a strongly dependent on:

1. Relative fiber, matrix, and interphase stiffness

2. Relative matrix and interphase strength

3. Interphase thickness (not varied in our calculations)

Here we consider both (1) and (2), and at present leave (3) for consideration in future work. In Figure 8 , the distance of the primary crack tip from the interphase/matrix interphase versus the ratio of the interphase to the matrix strength is given for both simulations with applied 2D plane strain and 3D axisymmetric boundary conditions. As can be seen in Figure 8, the distance of the primary crack from the interphase/matrix interface quickly approaches zero as the ratio of the interphase to matrix strength approaches 0.2 in the simulations performed here. It should be noted that secondary cracks initiated in the interphase when the primary crack tip was further away from the matrix-interphase boundary in the simulations with the applied 2D plane strain boundary conditions than in the simulations with 3D axisymmetric boundary conditions for the same ratio of the interphase to matrix strength.

Figure 9 shows the angle of the maximum principal stress on the plane of the primary crack at the interface as a function of the ratio of interphase to matrix strength for 3D axisymmetric simulations. Similar data is not given for the simulations with applied 2D plane strain boundary conditions because this angle was always $0^{\circ}$ when the secondary crack initiated with the primary crack tip far from the interphase and $90^{\circ}$ when the primary crack tip was at the matrix-interphase boundary. As can be seen in Figure 9, the ratio of the interphase to matrix strength approaches 0.2 as angle of the maximum principle stress relative to the interface begins to deviate from zero. These results corresponded to the observations regarding cases (1) and (2) described above associated with initiation of a single dominate secondary crack perpendicular to the interphase/matrix interface or multiple secondary cracking above and below the plane of the primary, respectively. Based on the simulations performed here, it appears that as the ratios of the 
interphase to matrix strength increasingly approach 0.2 , crack deflection is increasingly less certain. However, crack deflection is more likely when the interphase to matrix strength is less than 0.2. These conclusions hold for both the simulations with applied 2D plane strain and 3D axisymmetric boundary conditions.

For the simulations with applied 2D plain strain boundary conditions, two parameters were proposed to further quantify propensity for crack deflection in the case of secondary crack initiation (case a). These are

(i) the secondary crack leverage, or the horizontal distance from the secondary crack tip to the primary crack and

(ii) transition strain or the strain at the time of the transition of secondary crack into the matrix versus the interphase strength. Larger leverage is thought to indicate more prominent deflection; larger transition strain would indicate less prominent deflection.

Examination of Figures 10 and 11 that show the dependence of these parameters on coating strength indicate that both parameters are clearly separated into distinct groups by matrix strength, while the influence of the interphase strength on the damage response is much less. Comparison of Figures 10 and 11 confirms that larger leverage corresponds to lower transition strain and vice versa.

In contrast, the variety of cracking scenarios in 3D axisymmetric simulations complicates such quantification, in particular because the XFEM implementation in ABAQUS does not allow for crack coalescence. Specifically, when neighboring cracks grow to within one or two finite elements of each other they are not allowed numerically to combine and are forced to grow around each other; this results in artificially stiff regions near the point of intersection. A natural way to distinguish between different simulations would be to allow the simulations to progress until complete fracture; however, in the absence of crack coalescence the resulting artificially stiff regions and associated model prediction render subsequent parts of simulations to full fracture unreliable.

While the main material parameters that determined whether crack would deflect or penetrate were determined to be relative strengths of the matrix and interphase, the fracture energy of the interphase was very important in several cases. In particular, the only difference between the simulations shown in Figures 4 and 5 is the fracture energy of the interphase. With smaller fracture energy $\left(5 \mathrm{~J} / \mathrm{m}^{2}\right)$, the primary crack grows into the fiber with secondary cracks appearing near the fiber/interphase interface. With the fracture energy $15 \mathrm{~J} / \mathrm{m}^{2}$, the primary crack turns near the fiber/interphase interface without penetrating it. Increasing the fracture energy to $30 \mathrm{~J} / \mathrm{m}^{2}$, results in the primary crack penetrating the fiber. In this case, secondary cracks initiate in the interphase near the matrix/interphase interface, but are not in the path of the primary crack. In other simulations, the influence of the fracture energy of the interphase is less drastic. Such behavior was never observed in the simulations with applied plane strain boundary conditions.

\section{Conclusions}

A numerical study of the classical problem of propagation/deflection of a crack approaching an interface of dissimilar materials presented is quite complex. Unlike classical approaches, our 
numerical study allowed for crack initiation. This, for the chosen set of mechanical parameters, resulted in crack deflection at an interface dominated by secondary crack initiation inside the interface before the arrival of the primary crack's tip for both 2D and axisymmetric problem settings. This is, likely, the main mechanism of crack deflection in SiC/SiC CMCs where properties of fibers and matrix are comparable, while the interphase between them is significantly weaker.

Axisymmetric boundary conditions lead to results that are more in line with what has been observed experimentally and demonstrated by other modeling approaches. The results from the axisymmetric modes are more realistic and exhibit more varied crack configurations than previous studies demonstrated with applied 2D plane strain boundary conditions. For the chosen parameter set representing the melt-infiltrated SiC/SiC CMC HiPerComp®, the parameters having the greatest influence on the primary crack interaction with the interphase are relative strengths of the matrix and the interphase. Unlike the previous results from plane strain analysis, the fracture energy of the interphase was found to be quite important in many cases of axisymmetric simulations.

\section{Acknowledgments}

This work was in part funded by the Air Force Office of Scientific Research, task \#14RX06COR with Dr. David Stargel as the program manager.

\section{References}

[1] F. Rebillat, J. Lamon, and A. Guette, "The concept of a strong interface applied to $\mathrm{SiC} / \mathrm{SiC}$ composites with a BN interphase," Acta Materialia, vol. 48, pp. 4609-4618, 2000.

[2] M. Y. He and J. W. Hutchinson, "Crack deflection at an interface between dissimilar elastic materials," International Journal of Solids and Structures, vol. 25, pp. 1053-1067, 1989.

[3] D. Martinez and V. Gupta, "Energy criterion for crack deflection at an interface between two orthotropic media," Journal of the Mechanics and Physics of Solids, vol. 42, pp. 1247-71, 1994.

[4] M. Y. He, A. G. Evans, and J. W. Hutchinson, "Crack Deflection at an interface between dissimilar elastic materials: Role of residual stresses," International Journal of Solids and Structures, vol. 31, pp. 3443-3455, 1994.

[5] D. L. Tullock, I. E. Reimanis, A. L. Graham, and J. J. Petrovic, "Deflection and penetration of cracks at an interface between two dissimilar materials," Acta Metallurgica et Materialia, vol. 42, pp. 3245-52, 1994.

[6] B. K. Ahn, W. A. Curtin, T. A. Parthasarathy, and R. E. Dutton, "Criteria for crack deflection/penetration criteria for fiber-reinforced ceramic matrix composites," Composites Science and Technology, vol. 58, pp. 1775-1784, 1998.

[7] E. Martin, D. Leguillon, and C. c. Lacroix, "A revisited criterion for crack deflection at an interface in a brittle bimaterial," Composites Science and Technology, vol. 61, pp. 16711679, 2001. 
[8] H. Ming-Yuan, A. Bartlett, A. G. Evans, and J. W. Hutchinson, "Kinking of a crack out of an interface: role of in-plane stress," Journal of the American Ceramic Society, vol. 74, pp. 767-71, 04/ 1991.

[9] D. Xie, A. M. Waas, K. W. Shahwan, J. A. Schroeder, and R. G. Boeman, "Computation of energy release rates for kinking cracks based on virtual crack closure technique," CMES - Computer Modeling in Engineering and Sciences, vol. 6, pp. 515-524, 2004.

[10] D. Xie, A. M. Waas, K. W. Shahwan, J. A. Schroeder, and R. G. Boeman, "Fracture criterion for kinking cracks in a tri-material adhesively bonded joint under mixed mode loading," Engineering Fracture Mechanics, vol. 72, pp. 2487-2504, 11// 2005.

[11] E. Martin, P. W. M. Peters, D. Leguillon, and J. M. Quenisset, "Conditions for matrix crack deflection at an interface in ceramic matrix composites," Switzerland, 1998, pp. 291-302.

[12] T. A. Parthasarathy, G. J. Jefferson, and R. J. Kerans, "Analytical evaluation of hybrid ceramic design concepts for optimized structural performance," Materials Science and Engineering: A, vol. 459, pp. 60-68, 2007.

[13] P. F. Liu and Y. H. Yang, "Finite Element Analysis of the Competition Between Crack Deflection and Penetration of Fiber-Reinforced Composites Using Virtual Crack Closure Technique," Applied Composite Materials, pp. 1-13, 2014.

[14] P. S. Theocaris and J. Milios, "Disruption of a longitudinal interface by a moving transverse crack," Journal of Reinforced Plastics and Composites, vol. 2, pp. 18-28, 1983.

[15] N. J. Pagano and H. W. Brown, III, "The full-cell cracking mode in unidirectional brittlematrix composites," Composites, vol. 24, pp. 69-83, 1993.

[16] W. Lee, S. J. Howard, and W. J. Clegg, "Growth of interface defects and its effect on crack deflection and toughening criteria," Acta Materialia, vol. 44, pp. 3905-3922, 1996.

[17] Y. Kagawa and K. Goto, "Direct observation and modelling of the crack-fibre interaction process in continuous fibre-reinforced ceramics: model experiment," Materials Science and Engineering: A, vol. 250, pp. 285-290, 1998.

[18] J. Cook and J. E. Gordon, "A mechanism for the control of crack propagation in all-brittle systems," Proceedings of the Royal Society of London. Series A, Mathematical and Physical Sciences, vol. 282, pp. 508-520, 1964.

[19] D. Leguillon, C. Lacroix, and E. Martin, "Interface debonding ahead of a primary crack," Journal of the Mechanics and Physics of Solids, vol. 48, pp. 2137-2161, 2000.

[20] C. Lacroix, D. Leguillon, and E. Martin, "The influence of an interphase on the deflection of a matrix crack in a ceramic-matrix composite," Composites Science and Technology, vol. 62, pp. 519-523, 2002.

[21] E. Martin and D. Leguillon, "Energetic conditions for interfacial failure in the vicinity of a matrix crack in brittle matrix composites," International Journal of Solids and Structures, vol. 41, pp. 6937-6948, 2004.

[22] S. Pompidou and J. Lamon, "Analysis of crack deviation in ceramic matrix xomposites and multilayers based on the Cook and Gordon mechanism," Composites Science and Technology, vol. 67, pp. 2052-2060, 2007.

[23] T. Belytschko and T. Black, "Elastic crack growth in finite elements with minimal remeshing," International Journal for Numerical Methods in Engineering, vol. 45, pp. 601-620, 1999.

[24] N. Moes and T. Belytschko, "Extended finite element method for cohesive crack growth," Engineering Fracture Mechanics, vol. 69, pp. 813-833, 2002. 
[25] A. Hansbo and P. Hansbo, "A finite element method for the simulation of strong and weak discontinuities in solid mechanics," Computer Methods in Applied Mechanics and Engineering, vol. 193, pp. 3523-3540, 2004.

[26] "ABAQUS," 6.13 ed. Providence, RI, USA: Simulia, 2013.

[27] M. Braginsky and C. P. Przybyla, "Crack propagation/deflection in the interphase of $\mathrm{SiC} / \mathrm{SiC}$ ceramic matrix continuous fiber reinforced composites," in 19th International Conference on Composite Materials (ICCM), Montreal, Canada, 2013.

[28] M. Braginsky and C. P. Przybyla, "Dependence of crack propagation/deflection mechanism on characteristics of fiber coating or interphase in ceramic matrix continuous fiber reinforced composites," in 16th European conference on composite materials, Seville, Spain, 2014.

[29] D. Dunn, "The Effect of Fiber Volume Fraction in HiPerComp® SiC-SiC Composites," $\mathrm{PhD}$, Ceramics, Alfred University, Alfred, New York, 2010.

[30] "ABAQUS," 6.12-1 ed. Providence, RI, USA: Simulia, 2012.

[31] D. B. Marshall, P. E. Morgan, and R. M. Housley, "Debonding in multilayered composites of zirconia and LaPO4," Journal of the American Ceramic Society, vol. 80, pp. 1677-1683, 1997.

[32] A. G. Evans, "Overview No. 125 Design and life prediction issues for high-temperature engineering ceramics and their composites," Acta Materialia, vol. 45, pp. 23-40, 1997.

[33] A. G. Evans and D. B. Marshall, "Overview no. 85 The mechanical behavior of ceramic matrix composites," Acta Metallurgica, vol. 37, pp. 2567-2583, 1989.

[34] A. G. Evans, "The mechanical performance of fiber-reinforced ceramic matrix composites," Materials Science and Engineering: A, vol. 107, pp. 227-239, 1989.

Tables

Table 1. Properties of HiPerComp® SiC-SiC CMCs [29].

\begin{tabular}{l}
\hline \multicolumn{1}{c}{ Matrix } \\
\hline Elastic properties $\mathrm{E}=360 \mathrm{GPa}, v=0.185$ \\
Initiation stress $\sigma_{\mathrm{I}}=0.8 \mathrm{GPa}$ (maximum principal) \\
Fracture energy $36 \mathrm{~J} / \mathrm{m}^{2}$ \\
Tolerance $0.05 ;$ Damage stabilization 0.005 \\
\hline Interphase (BN) \\
\hline Elastic properties $\mathrm{E}=10 \mathrm{GPa}, v=0.05$ \\
Initiation stress $\sigma_{\mathrm{I}}=75 \mathrm{MPa}$ (maximum principal) \\
Fracture energy $5 \mathrm{~J} / \mathrm{m}^{2}$ \\
Tolerance $0.05 ; \mathrm{Damage}$ stabilization 0.01 \\
\hline \multicolumn{1}{c}{ Fiber } \\
Elastic properties $\mathrm{E}=380 \mathrm{GPa}, v=0.185$ \\
Initiation stress $\sigma_{\mathrm{I}}=2.6 \mathrm{GPa}$ (maximum principal) \\
Fracture energy $50 \mathrm{~J} / \mathrm{m}^{2}$ \\
Tolerance $0.05 ;$ Damage stabilization 0.005 \\
\hline
\end{tabular}

Table 2. Variation of damage parameters employed in the simulations. Parameters employed in the simulations with 2D plane strain boundary conditions did not include those indicated by an 
asterisk. The simulations with 3D axisymmetric boundary conditions were performed for all the listed conditions.

\begin{tabular}{ccc}
\hline & $\boldsymbol{\sigma}_{\mathbf{I}}$ & $\begin{array}{c}\text { Fracture } \\
\text { energy }\end{array}$ \\
\hline Matrix & & \\
& $0.4 \mathrm{GPa}$ & $36 \mathrm{~J} / \mathrm{m}^{2}$ \\
& $0.8 \mathrm{GPa}$ & \\
$1.2 \mathrm{GPa}$ & \\
\hline Interphase & & \\
& $50 \mathrm{MPa}$ & $5 \mathrm{~J} / \mathrm{m}^{2}$ \\
& $75 \mathrm{MPa}$ & $10 \mathrm{~J} / \mathrm{m}^{2}$ \\
& $100 \mathrm{MPa}$ & $15 \mathrm{~J} / \mathrm{m}^{2}$ \\
& $150 \mathrm{MPa}$ & $* 20 \mathrm{~J} / \mathrm{m}^{2}$ \\
& ${ }^{*} 200 \mathrm{MPa}$ & ${ }^{*} 30 \mathrm{~J} / \mathrm{m}^{2}$ \\
& $300 \mathrm{MPa}$ & \\
\hline Fiber & $2.6 \mathrm{GPa}$ & $50 \mathrm{~J} / \mathrm{m}^{2}$ \\
\hline
\end{tabular}

\section{Figures}

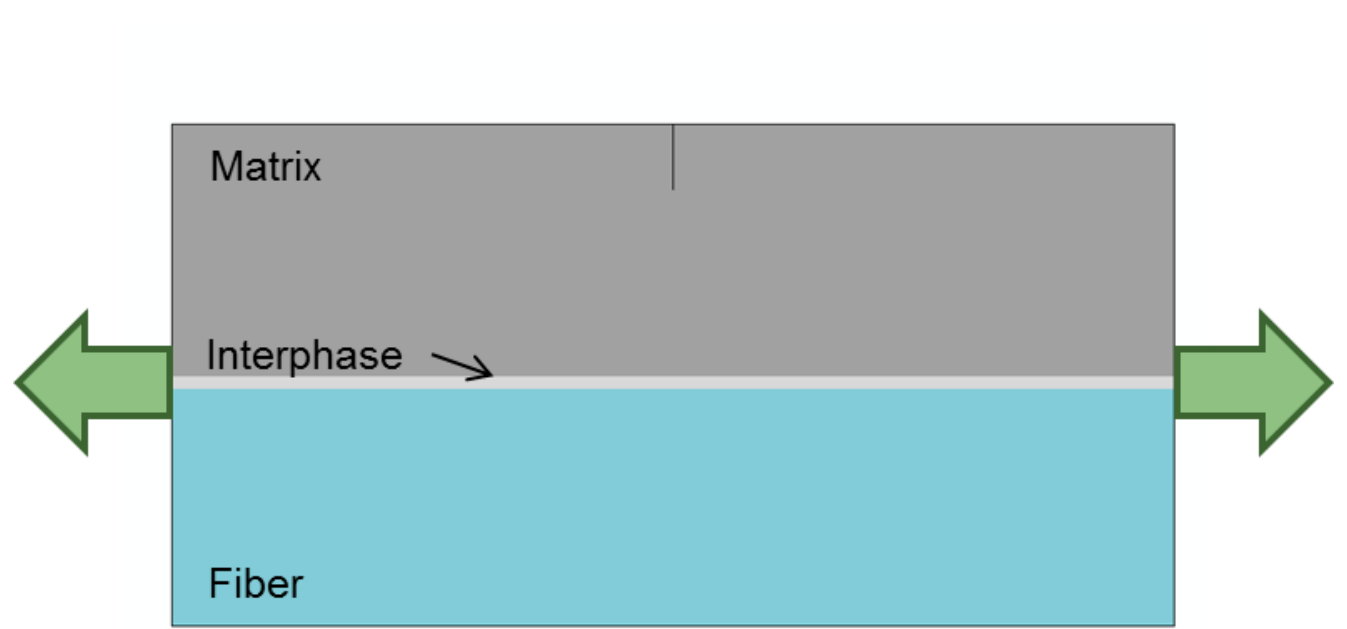

Figure 1. Matrix-Interphase-Fiber model with applied 2D plane strain boundary conditions with pre-crack. 

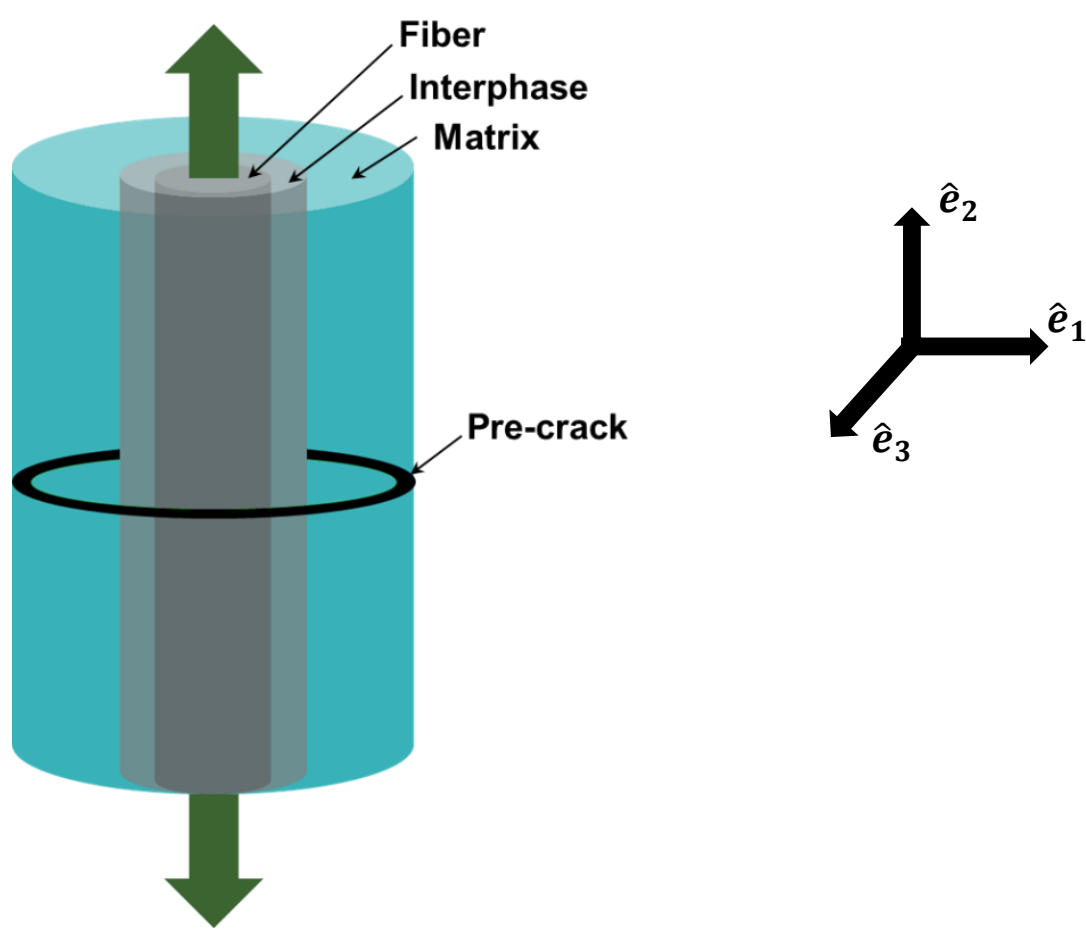

Figure 2. Matrix-Interphase-Fiber model with applied 3D axisymmetric boundary conditions with pre-crack. 


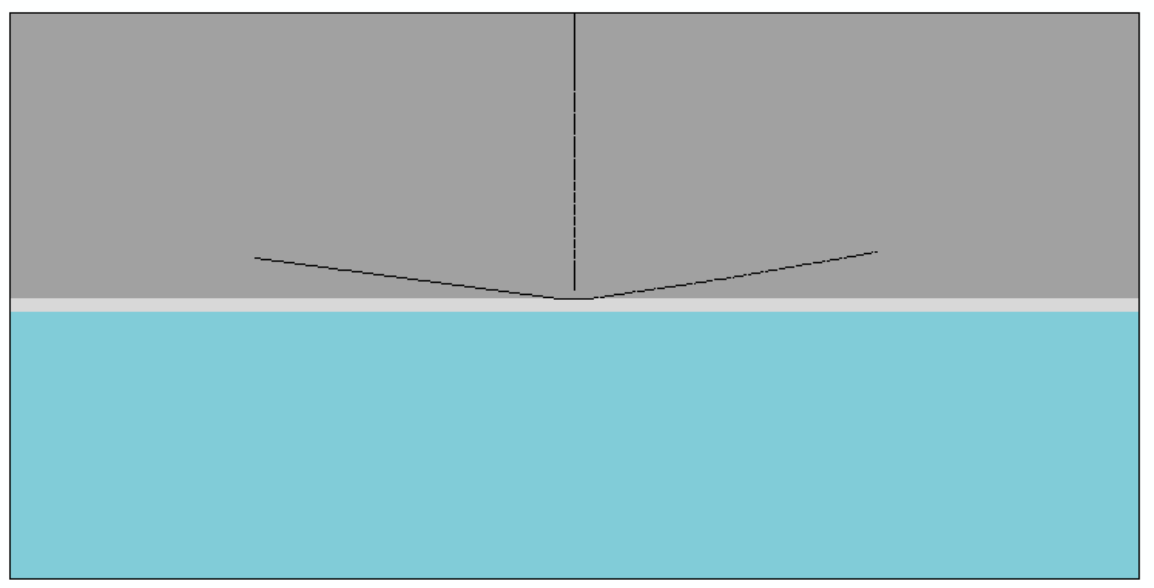

(a)

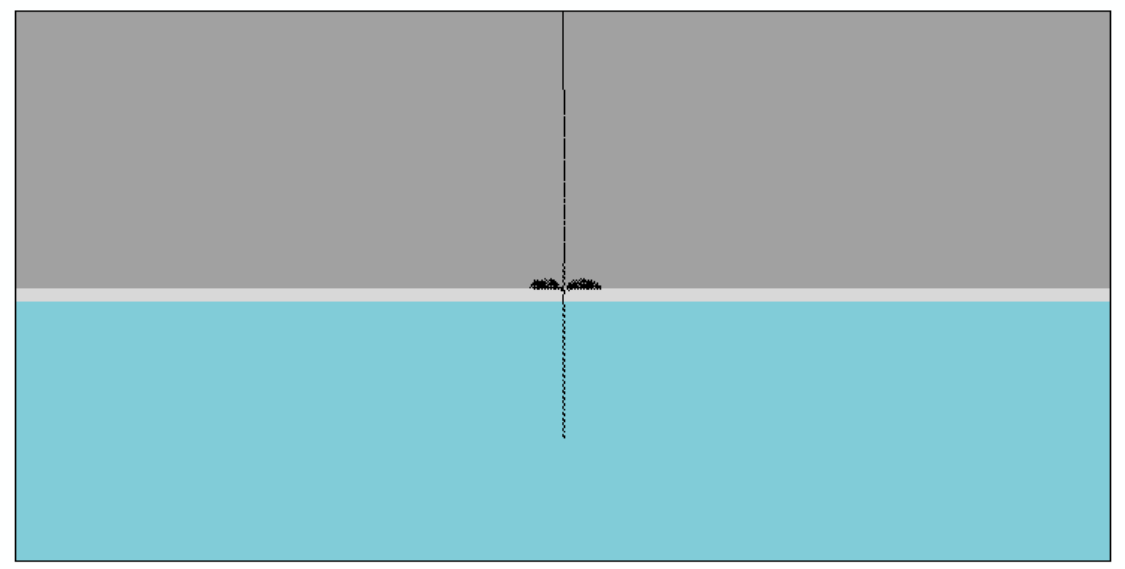

(b)

Figure 3. Two distinct mechanisms are observed with 2D plane strain boundary conditions: (a) deflection; (b) penetration 


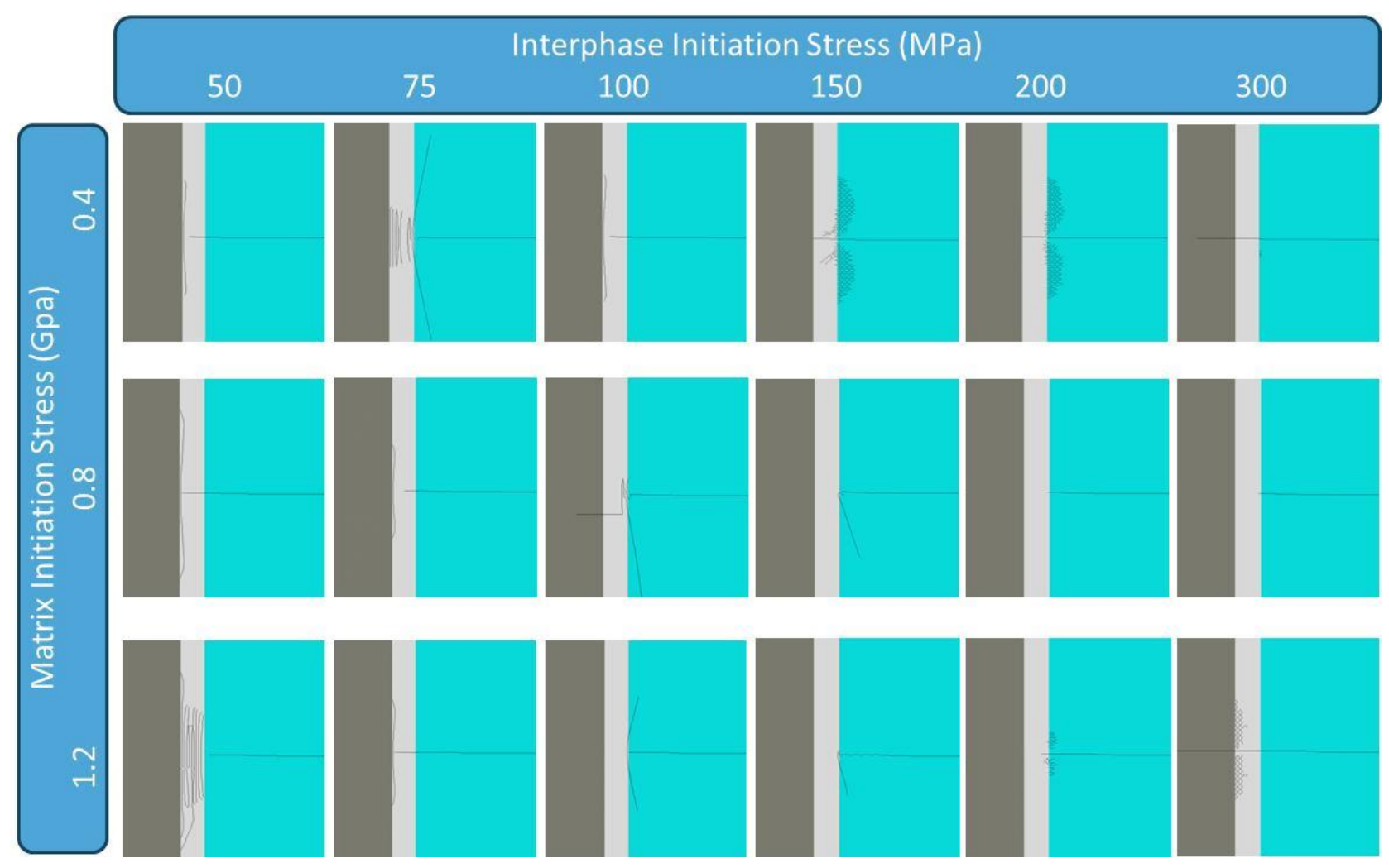

Figure 4. Variety of crack patterns observed for simulations with 3D axisymmetric boundary conditions and interphase fracture energy of $5 \mathrm{~J} / \mathrm{m}^{2}$. 


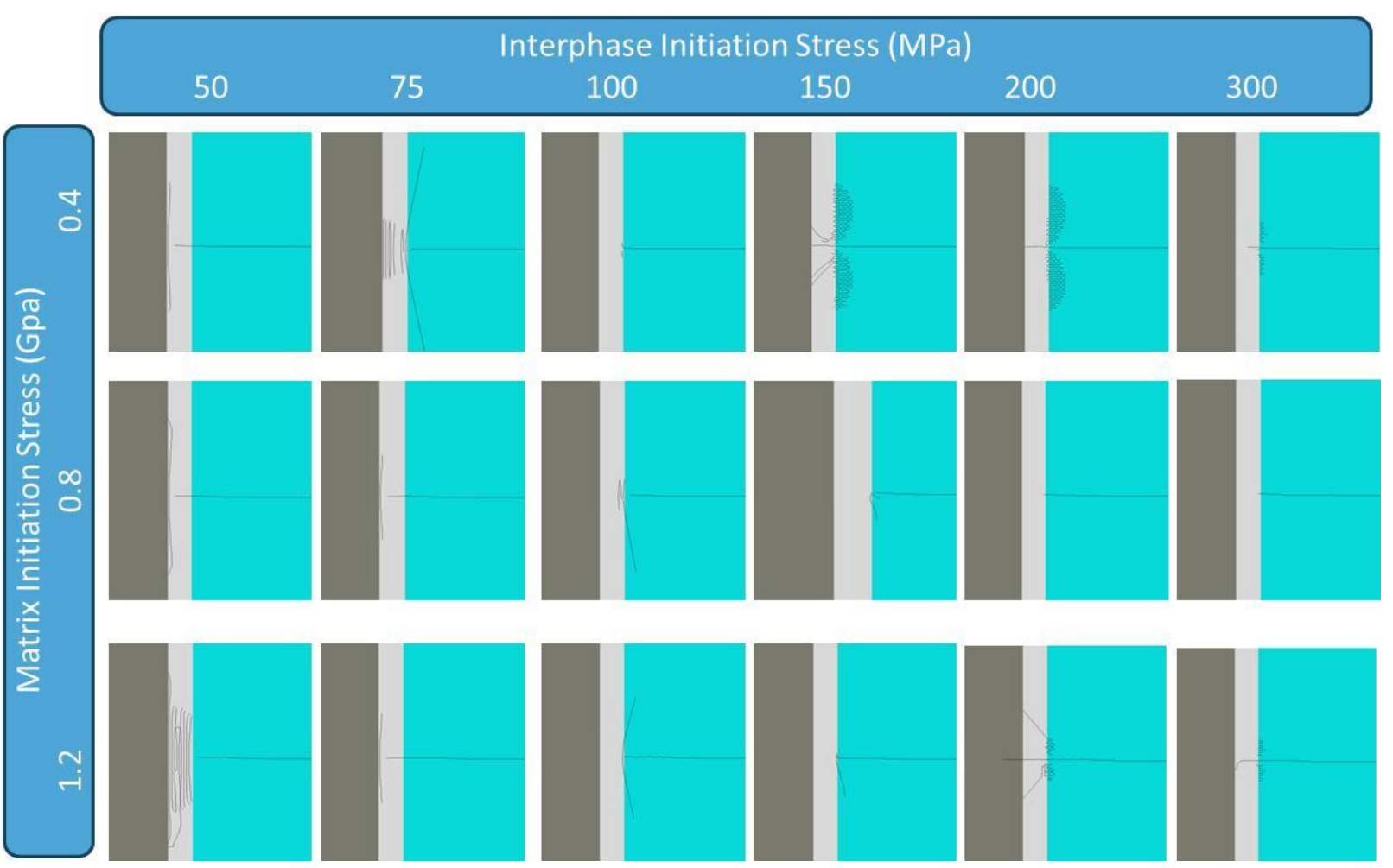

Figure 5. Variety of crack patterns observed for simulations with 3D axisymmetric boundary conditions and interphase fracture energy of $15 \mathrm{~J} / \mathrm{m}^{2}$. 

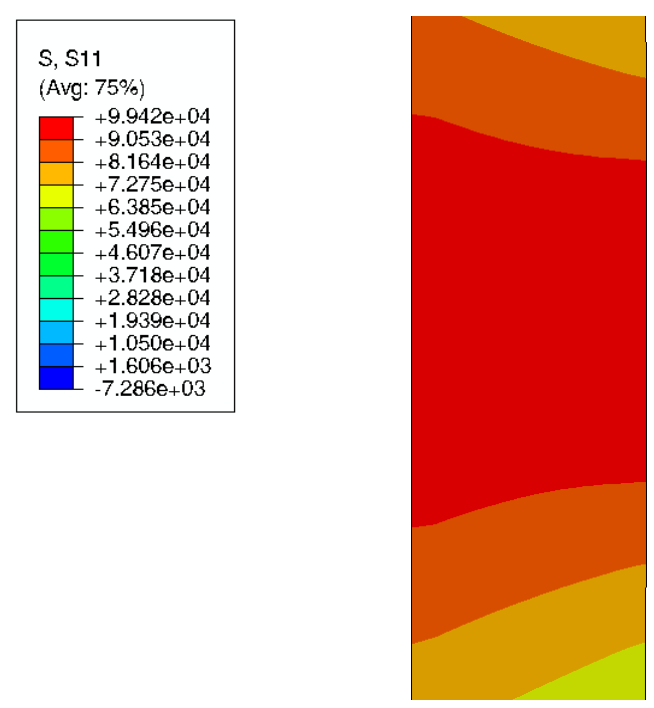

(a)

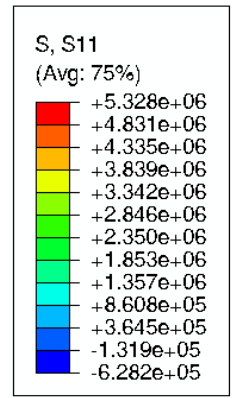

Interphase

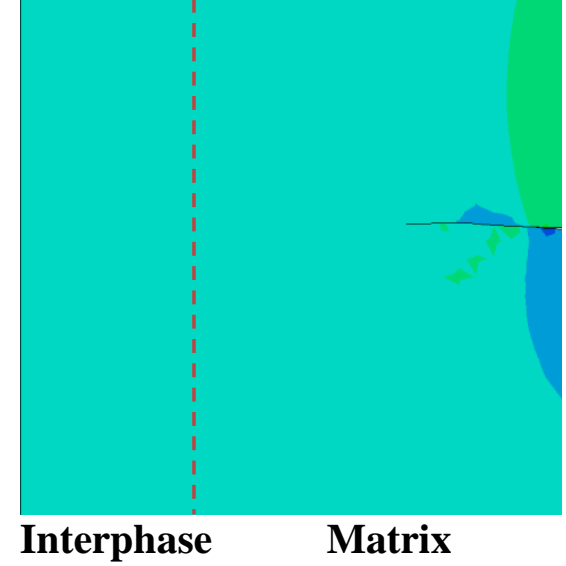

(c)

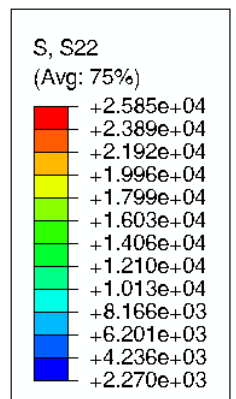

(b)

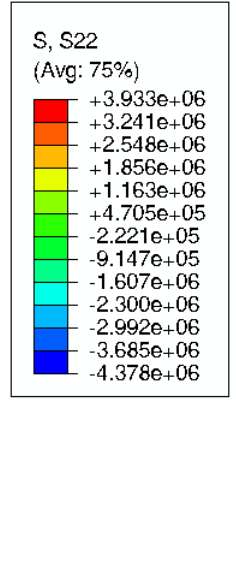

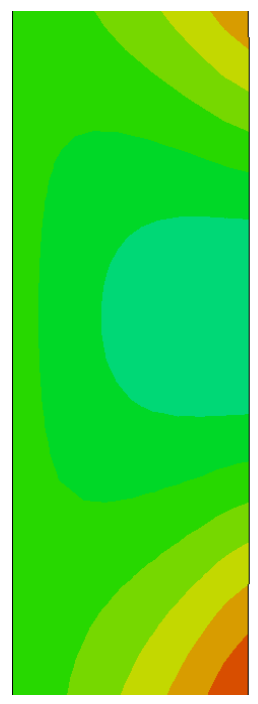

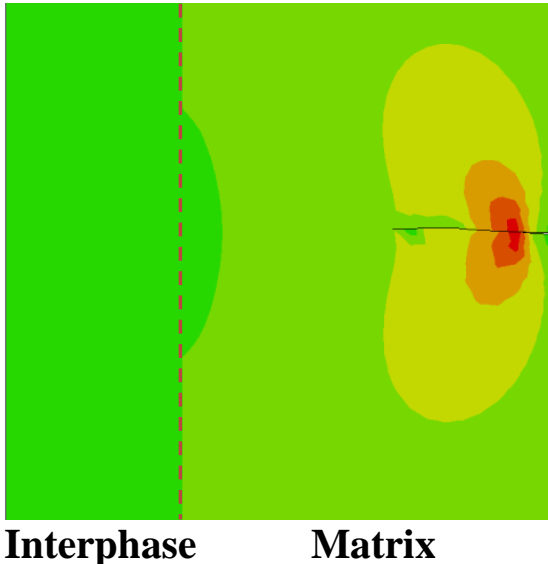

(d)

Figure 6. Normal components of the stress field inside the interphase before the secondary cracking in the interphase for an axisymmetric simulation with primary crack tip at a distance away from the interphase/matrix interface on the order of several times the interphase thickness; (a) $\sigma_{11}$ in the interphase, (b) $\sigma_{22}$ in the interphase, (c) $\sigma_{11}$ in the matrix and interphase, (d) $\sigma_{22}$ in the matrix and interphase. Note the dotted lines in (c) and (d) indicate the interphase/matrix interface. 

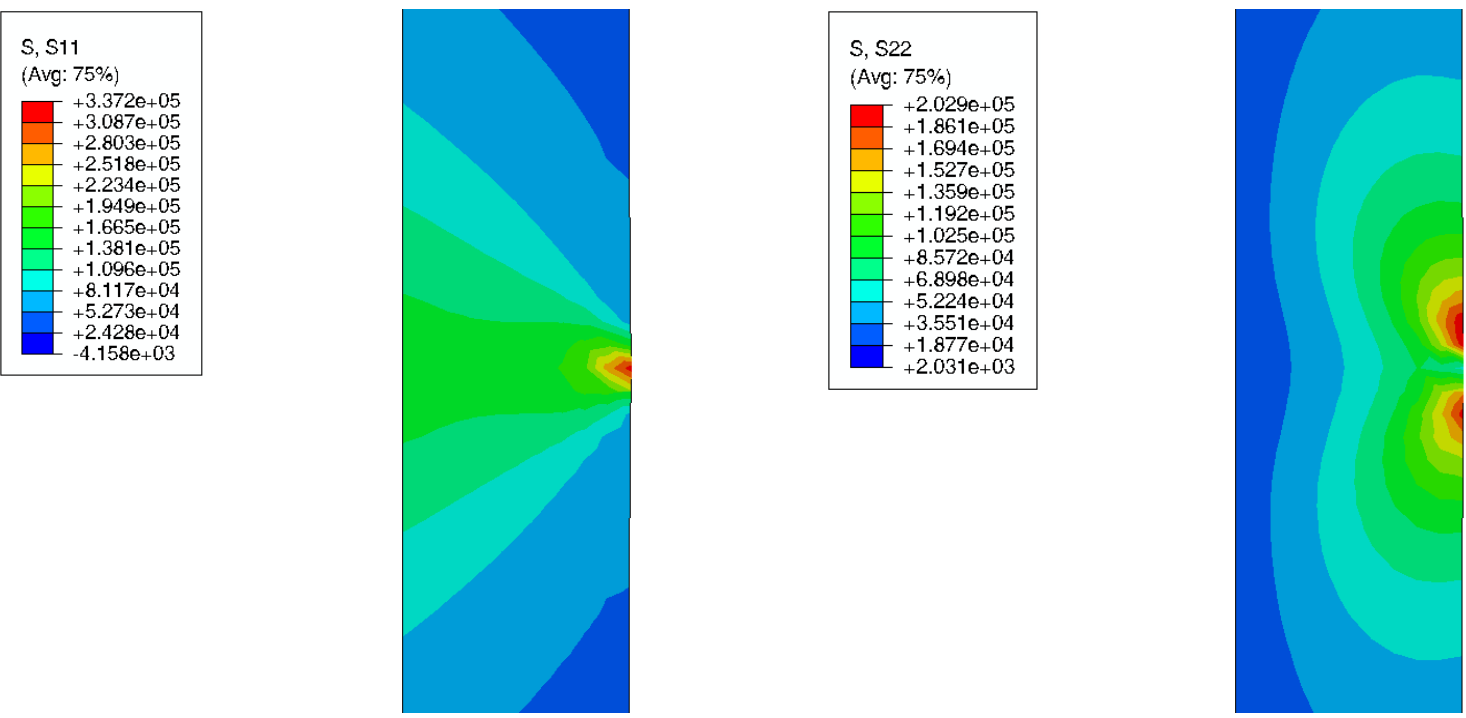

(a)

(b)
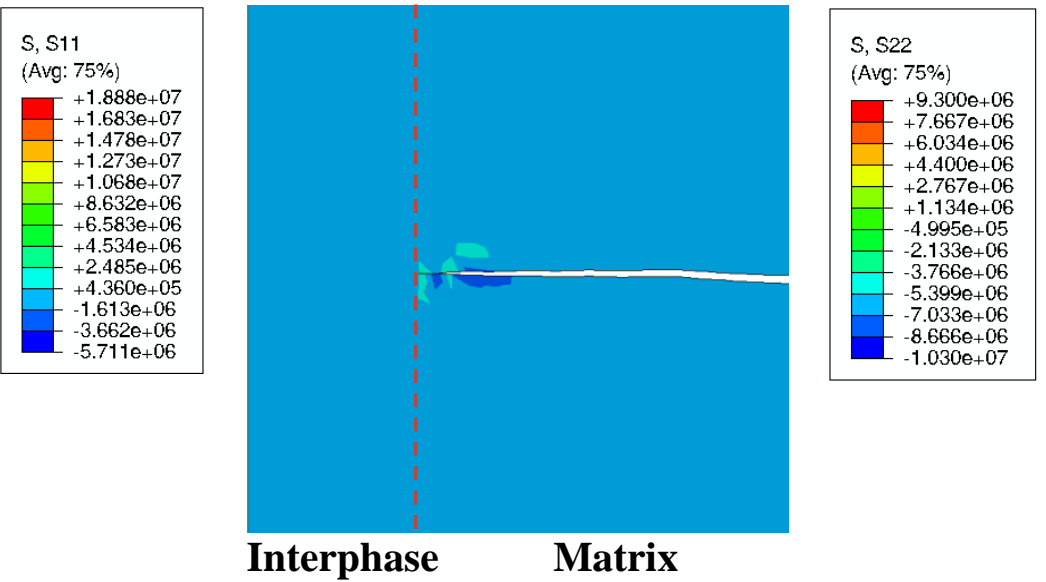

(c)

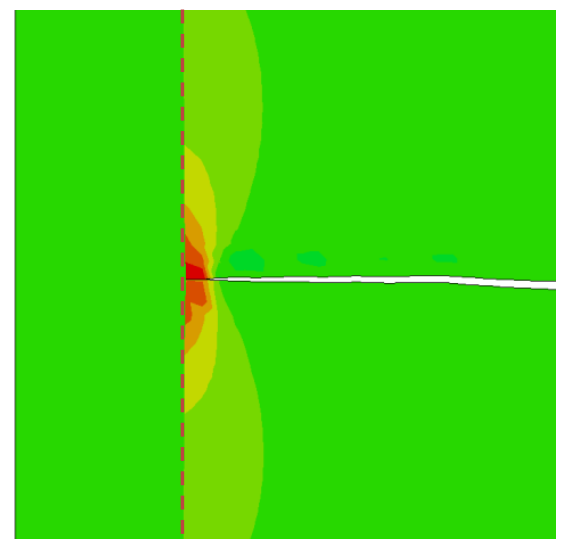

Interphase Matrix

(d)

Figure 7. Normal components of the stress field inside the interphase before secondary crack initiation in the interphase for an axisymmetric simulation with the primary crack tip intersecting the interphase/matrix interface; (a) $\sigma_{11}$ in the interphase, (b) $\sigma_{22}$ in the interphase, (c) $\sigma_{11}$ in the matrix and interphase, (d) $\sigma_{22}$ in the matrix and interphase. Note the dotted lines in (c) and (d) indicate the coating/matrix interface. 


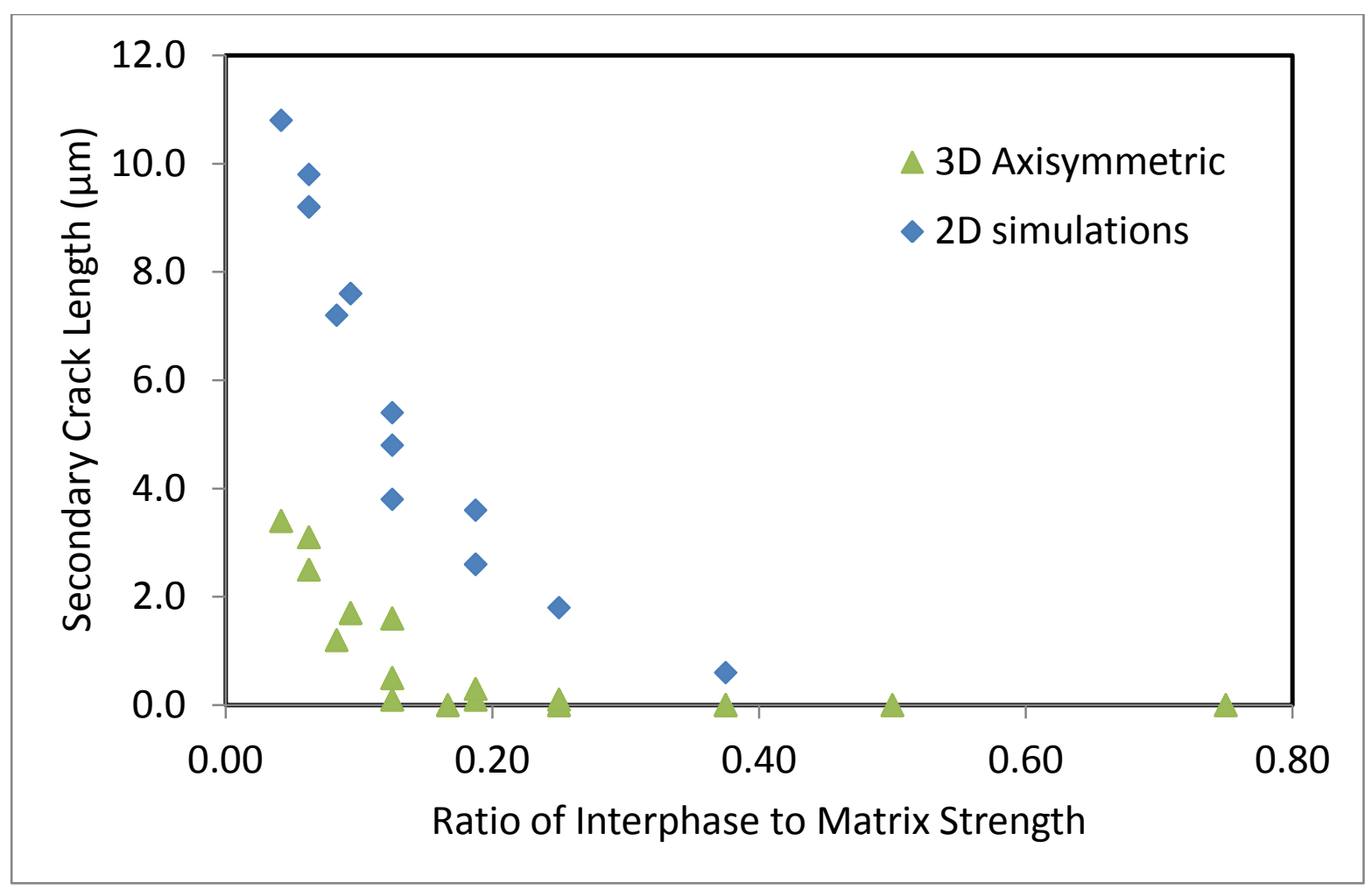

Figure 8 . Distance of the primary crack tip from the interphase/matrix interface, immediately prior to secondary crack initiation in the interphase as a function of the ratio of the interphase to matrix strength. 
7

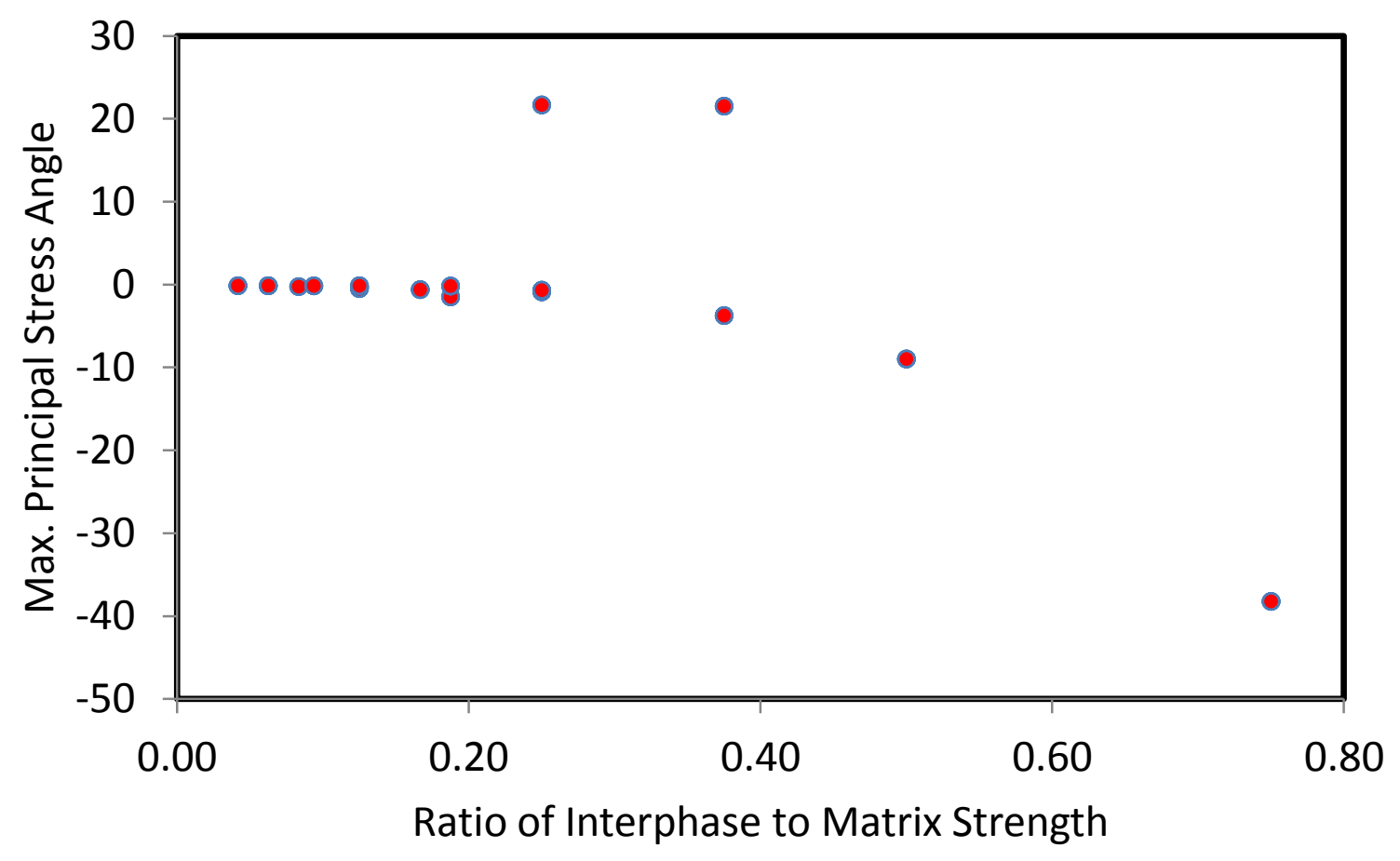

Figure 9. Angle of the maximum principal stress as a function of the ratio of interphase to matrix strength. 


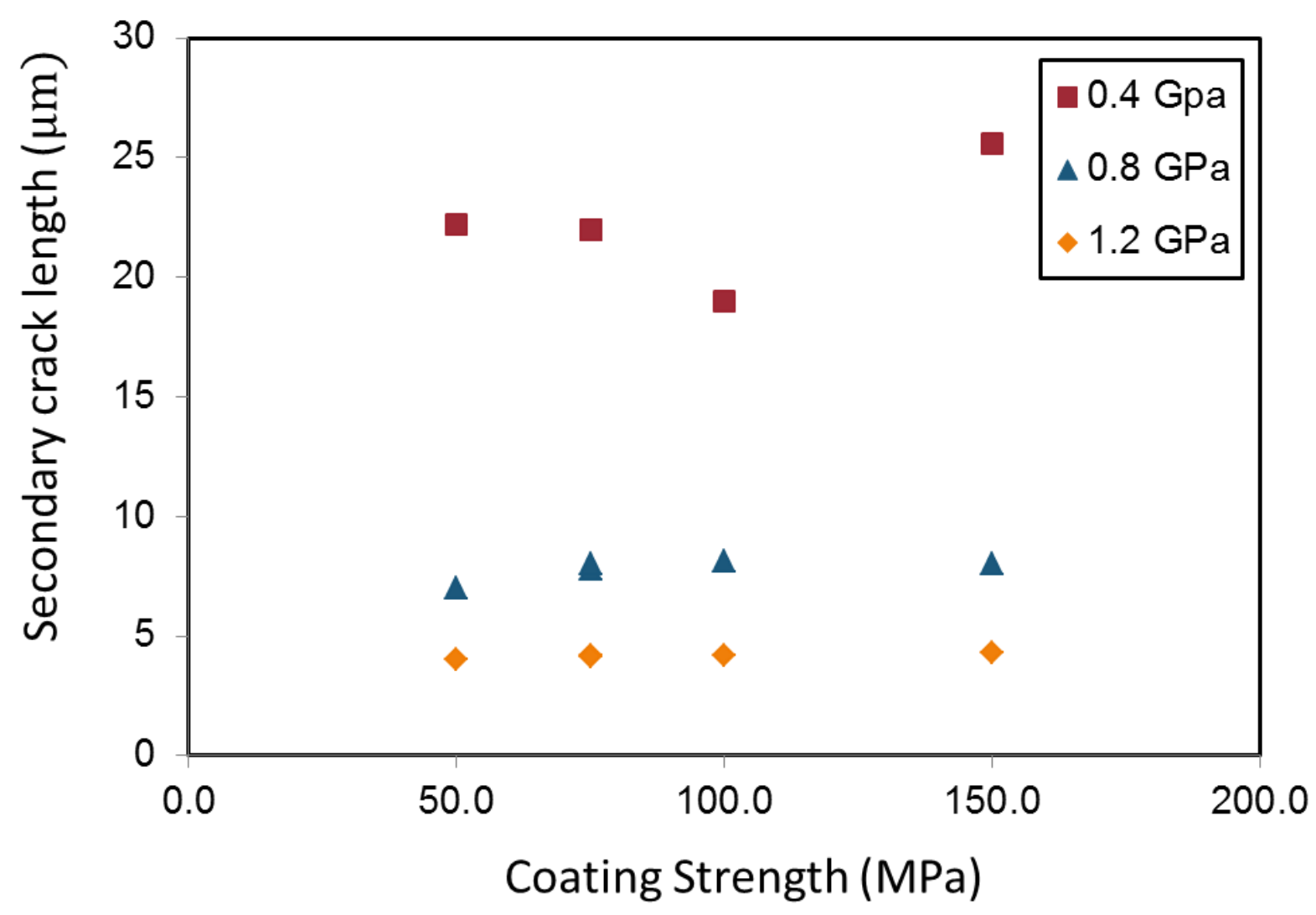

Figure 10. Secondary crack length at time of intersection with primary crack versus interphase strength for three different matrix strengths as indicated in the legend for the simulations with 2D plane strain boundary conditions 


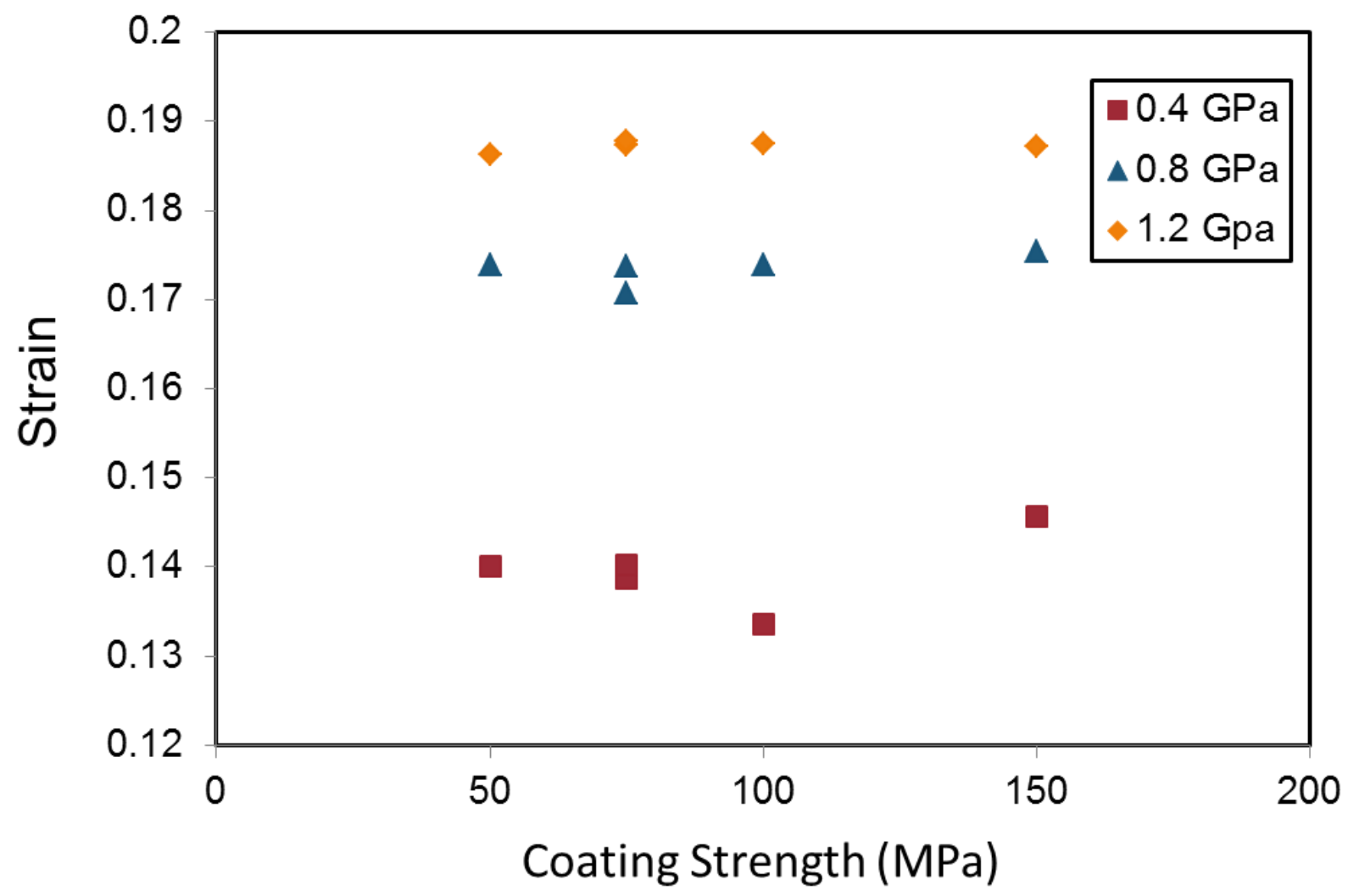

Figure 11. Transition strain - strain at the time of transition of secondary crack into the matrix versus the interphase strength for three different matrix strengths and indicated in the legend for the simulations with 2D plane strain boundary conditions. 